\title{
Photodynamic Therapy- A Ray towards Periodontics
}

\author{
Dr. Dheeraj Khurana ${ }^{1}$, Dr. Sharnamma B. ${ }^{2}$, Dr. Promod Kumar Rathore ${ }^{3}$, \\ Dr. Prashant Tyagi ${ }^{3}$ \\ ${ }^{I}$ (P.G Student, Dept. of Periodontics, Shree BankeyBihari Dental college, Ghaziabad, India) \\ ${ }^{2}$ (Professor and Head, Dept. of Periodontics, Shree BankeyBihari Dental college, Ghaziabad, India) \\ ${ }^{3}$ (Sr. lecturer, Dept. of Periodontics, Shree BankeyBihari Dental college, Ghaziabad, India)
}

\begin{abstract}
The increase of microbial resistance and development of lighting devices with safe and accurate delivery of energy, today professionals can count on therapies to manage pathogens in different fields of dentistry, especially periodontics. The possibility of development of resistance to antibiotics by the target organism has led to development of a new concept with fewer complications i.e. Photodynamic Therapy. The combination of laser light and photosensitizer known as photodynamic therapy (PDT) can be used in periodontal therapy. LASER produces a monochromatic, collimated and coherent light beam. This unique form of light can interact with living tissues leading to surgical and/ or therapeutic applications. A Photosensitizer is a dye substance that is absorbed by the microorganism, cell or tissue allowing it to interact with the light. This mode of treatment is referred to as photodynamic therapy (PDT) or photo-activated disinfection (PAD). Application of photodynamic therapy in periodontics such as pocket debridement, gingivitis, aggressive periodontitis continue to evolve into a mature clinical treatment modality, and is considered as a promising novel approach for eradicating pathogenic bacteria in periodontitis. This Article presents an overview of photodynamic therapy as it represents a novel therapeutic approach in the management of periodontal diseases.
\end{abstract}

Keywords: Light, Periodontitis, Peri-Implantitis, Photoactivated Disinfection, Photodynamic Therapy,

Photosensitizing agents.

\section{Introduction}

The main objective of periodontal therapy is to eliminate deposits of bacteria and bacterial niches by removing the supragingival and subgingival biofilm. In the treatment of periodontally involved teeth, current concepts are based on mechanical scaling and root planing to remove bacterial deposits, calculus, and cementum contaminated by bacteria and endotoxins. Some therapeutic alternatives, such as systemic and local antibiotics, have been used in cases not responding to conventional treatments, although this therapy brings undesirable side effects like the emergence of resistant microorganisms and a shift in the microflora after extended use which limit the use of antimicrobials. ${ }^{2}$

Recent advances in technology have led to a constant drive to develop novel approaches for the treatment of periodontal diseases. The need to find more optimal treatment protocols for periodontal disease is a long-term goal for periodontal researchers and clinicians. A novel noninvasive photochemical approach for infection control, namely photodynamic therapy, has been receiving much attention in the treatment of oral diseases. ${ }^{1}$ Photodynamic therapy (PDT), also known as photoradiation therapy, phototherapy, or photochemo therapy. ${ }^{3}$ Photodynamic therapy (PDT) was introduced in medical therapy in 1904 as the light-induced inactivation of cells, microorganisms or molecules and is based on the principle that a photosensitizer (i.e. a photoactivatable substance) binds to the target cells and can be activated by light of a suitable wavelength in the presence of oxygen(Fig. 1) ${ }^{3,4}$ The exposure of the photosensitizer to light results in the formation of toxic oxygen species, causing localized photodamage and cell death. Clinically, this reaction is cytotoxic and vasculotoxic. ${ }^{5}$ The oral cavity is especially suitable for photodynamic therapy (PDT) because it is relatively accessible to illumination. ${ }^{3,5}$ This review is aimed to discuss the role of PDT in periodontal therapy.

Figure 1.Factors for successful photodynamic therapy

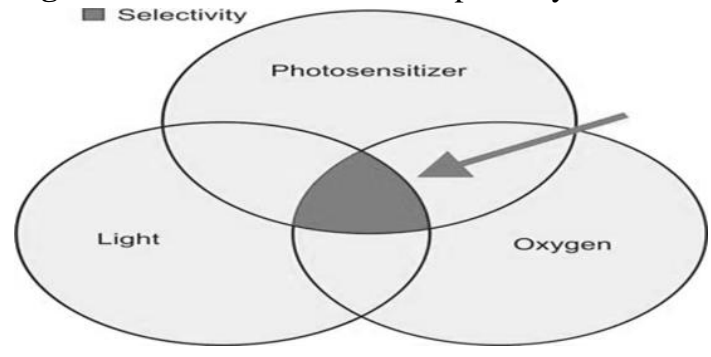




\section{History Behind Photodynamic Therapy}

The concept of treatment with light and photoactive compounds can be traced back over 6000 years to the ancient Egyptians who used light-sensitive substances (psoralens) by crushing leaves of plants related to parsley with sunlight to treat sunburns. Reference to the use of a plant extract for the restoration of skin pigmentation was made in $1400 \mathrm{BC}$ and phototoxic effects of psoralens were described in $1250 \mathrm{AD}$. Ancient Indians also believed that vitiligo could be treated by the combination of extracts of Psoraleacorylifolia and light ${ }^{6}$ but it disappeared for many centuries, only being rediscovered by the Western civilization at the beginning of the 20th century. ${ }^{2}$

The use of photodynamic therapy for inactivating microorganisms was first demonstrated more than 100 years ago, when Oscar Raab reported the lethal effect of acridine hydrochloride and visible light on Paramecia caudatum. ${ }^{7}$ In 1904, Jodlbaner and Von Tappeiner coined the term photodynamic to describe oxygen-dependent chemical reactions induced by photosensitization which could inactivate bacteria. ${ }^{8}$ The German physician Friedrich Meyer-Betz performed the pioneering study which was at first called photo radiation therapy (PRT) with porphyrins in 1913. He tested the effects of hematoporphyrin-PRT on his own skin. ${ }^{9}$ In 1920s Policard noted that tumor tissue was inherently more fluorescent than healthy tissue. ${ }^{10}$ It was John Toth, as product manager for Cooper Medical Devices Corp/Cooper Lasersonics, who acknowledged the "photodynamic chemical effect" of the therapy with early clinical argon dye lasers and wrote the first "white paper" renaming the therapy as "Photodynamic Therapy" (PDT). ${ }^{9,11,12}$ In 1950s Ronchese attempted to activate endogenous fluorescent molecules in tumor tissue to delineate its boundaries more accurately. In the 1960s Winkelman used synthetic porphyrins (tetraphenylporphines) to detect tumor tissue. In 1976; Weisshaupt et al identified the cytotoxic product of photochemical reaction to be singlet oxygen ${ }^{10}$ Kelly and Snell performed the first experiments in PDT on humans. They investigated effects of hematoporphyrin derivatives (HpD) photodynamic therapy in case of bladder carcinoma in five patients. ${ }^{13}$ In 1978, Daugherty et al. successfully applied this novel technique for the treatment of different cancers. It was thought that a common feature between tumour cells and micro-organisms was high proliferation and an active metabolism. Since microorganisms are able to accumulate different photosensitizers, it is believed that photodynamic inactivation of them might be effective. ${ }^{8}$ PDT was first approved by the Food and Drug Administration of U.S in 1999 to treat pre-cancerous skin lesions of the face or scalp. PDT has extensively been used to treat cancers and certain other diseases. PDT has emerged in recent years as a new non-invasive therapeutic modality. ${ }^{9}$

\section{Principles behind Pdt}

The knowledge of the preferred uptake and accumulation of some dyes (mostly porphyrins) into tumor tissues stimulated the introduction of PDT into clinical practice. ${ }^{14,17}$ Photodynamic therapy (PDT) is the lightinduced non-thermal inactivation of cells, microorganisms, or molecules. This utilizes light to activate a photosensitizing agent in the presence of oxygen. ${ }^{5}$ Upon irradiation with light of a specific wavelength, the photosensitizer undergoes a transition from a low-energy ground state to an excited singlet state. Subsequently, the photosensitizer may decay back to its ground state, with emission of fluorescence, or may undergo a transition to a higher-energy triplet state. The triplet state can react with endogenous oxygen to produce singlet oxygen and other radical species, causing a rapid and selective destruction of the target tissue. ${ }^{3,8,10}$ This reactive oxygen species (ROS) produced by a non-toxic dye or photosensitizer (PS) molecule (Fig. 2)can kill mammalian or microbial cells. ${ }^{16}$

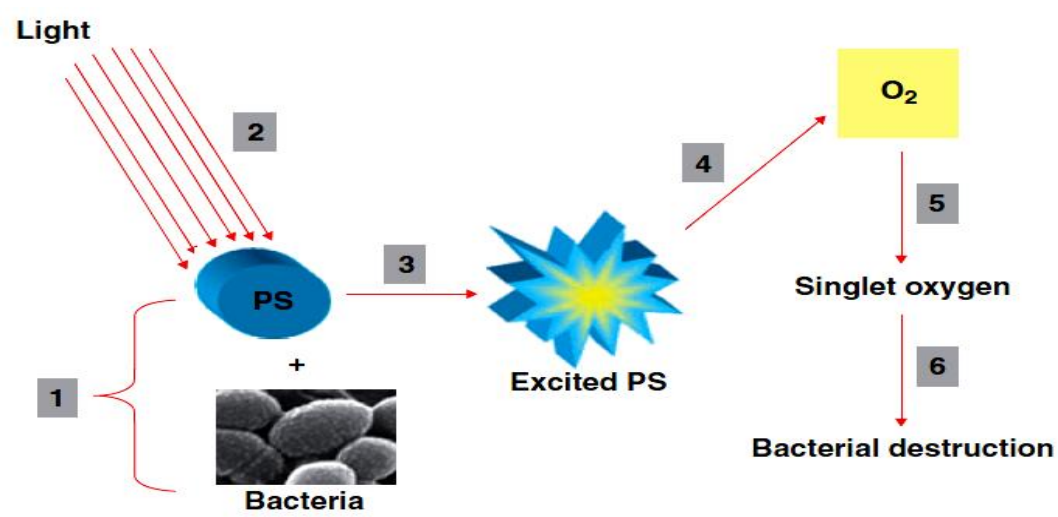

Figure 2.Mechanism of photodynamic therapy action. A photosensitizer is taken up by microorganisms (1) and following exposure to light of the appropriate wavelength (2) becomes activated to an excited state (3). Then, the photosensitizer transfers energy from light to molecular oxygen (4) to generate singlet oxygen and free radicals (5) that are cytotoxic to cells (6). ${ }^{7}$ 


\section{III .I Light}

Light has been used for therapeutic purposes for many centuries. Initially, ultraviolet radiation through sunlight and later using artificial UV light was applied in the treatment of acne vulgaris, skin tuberculosis and rickets. ${ }^{17}$

PDT requires a source of light that activates the photosensitizer by exposure to low-power visible light at a specific wavelength. Human tissue transmits red light efficiently, and the longer activation wavelength of the photosensitizer results in deeper light penetration. Consequently, most photosensitizers are activated by red light between 630 and $700 \mathrm{~nm}$ (Fig. 3), corresponding to a light penetration depth from $0.5 \mathrm{~cm}$ (at $630 \mathrm{~nm}$ ) to 1.5 $\mathrm{cm}(\text { at } \sim 700 \mathrm{~nm})^{3}$

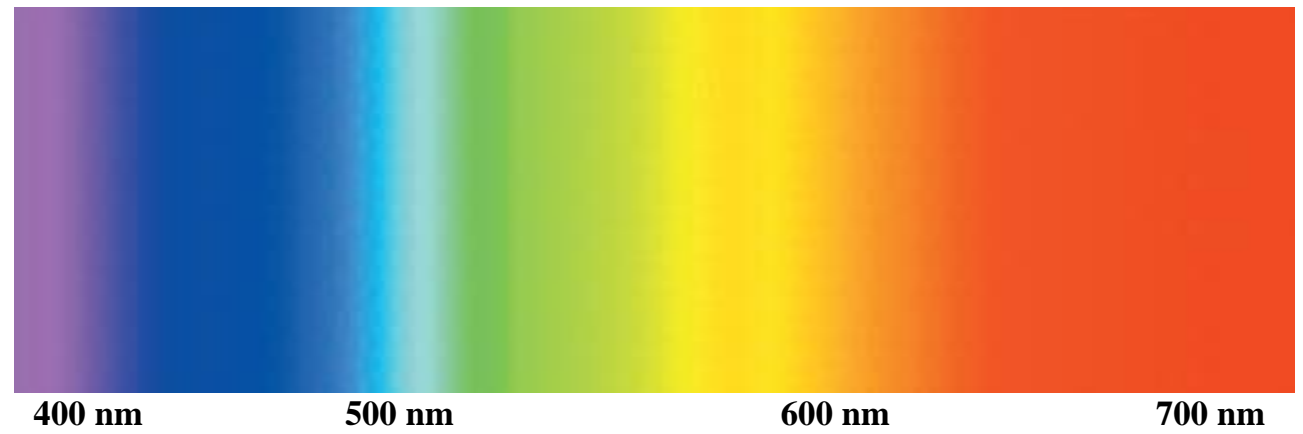

Figure 3.Visible light, which covers the range of 400-0700 $\mathrm{nm}$ of all electromagnetic radiation, is most relevant to photodynamic therapy. However, in practice, the range of light used in photodynamic therapy is generally

$>600 \mathrm{~nm}$. This is because endogenous molecules, such as hemoglobin, absorb light strongly at wavelengths of<600 nm and therefore capture most of the incoming photons. ${ }^{7}$

With regard to the application of subablative (non-surgical) low level laser photonic energy in clinical practice, several light-related phenomena have been adapted for therapeutic use:

- Photoluminescence: this may occur as a visual phenomenon (of use in photodynamic diagnosis)

- Chemodynamics: an administered photosensitizer acts as the source of subsequent fluorescence, initiating further energy transfer to adjacent tissue components. ${ }^{17}$

There are three light systems for the photodynamic therapy:

1.Diode laser systems: They are easy to handle, portable, and cost-effective.

2.Noncoherent light sources: Preferred for treatment of larger areas and include tungsten filament, quartz halogen, xenon arc, metal halide and phosphor-coated sodium lamps.

3.Nonlaser light sources include light emitting diodes (LED):They are economical, light weight, and highly flexible. ${ }^{10,18}$

The use of a non-ablation, low-level laser wavelength to initiate photodynamic antimicrobial chemotherapy through a suitably administered photosensitizer, may be seen to have the following advantages over 'conventional' laser use:

- Non-surgical (sub-ablative) photonic energy values are employed

- Primary interaction is indirect and through a chemical mediator (photosensitizer)

- $\quad$ Little risk of collateral damage within confined target sites

- The use of non-collimated light (emitting light at a variety of angles from the source) through a diffuser tip can overcome limited access and be further compensated by scatter through the body of the liquid sensitizer. ${ }^{17}$

Sources used for light delivery in PDT vary, depending upon the location and morphology of the lesion, but are typically fiber-optic catheters terminated with cylindrical diffusers or lenses for flat-field applications (Biel, 2002; Mang, 2004; Allison et al., 2005). The light field produced should be uniform, allowing for a precise calculation of the delivered dose. Devices that could simultaneously monitor both light delivery and sensitizer fluorescence would greatly advance PDT as a more routine clinical treatment (Gudgin Dickson et al., 2002). ${ }^{3}$

\section{III .II Photosensitive Agents:}

An ideal photosensitizer

a) Should be chemically pure and of known specific composition.

b) Should have a high quantum yield for singlet oxygen production.

c) Should have a strong absorption with high extinction coefficient $\mathrm{E}$ at longer wavelength (red) region preferably between $630-700 \mathrm{~nm}$.

d) Should have an excellent photochemical reactivity. 
e) Should possess minimal dark toxicity.

f) Only be toxic in the presence of light.

g) Should have preferable retention by target tissue.

h) Should be rapidly excreted from the body, and finally.

i) Should be synthesizable from easy available precursors and should be stable and easy to dissolve in the body's tissue fluids and be capable of formulation. ${ }^{19}$

Most of the photosensitizers under investigation for cancer PDT are based on the tetrapyrrole nucleus, such as porphyrins, chlorins, bacteriochlorins and phthalocyanines. The first approved photosensitizer was hematoporphyrin derivative (Photofrin; AxcanPharma Inc., Mont-Saint-Hilaire, Canada) for the treatment of refractory superficial bladder cancer. ${ }^{7,20}$

\section{III .II .I Types Of Photosensitizers:Based On The Following Basic Structures:}

- Tricyclic dyes (methylene blue, toludine blue O, and acridine orange) (2) Phthalocyanines (aluminum disulfonatedphthalocyanine and cationic $\mathrm{Zn}$ (II) phthalocyanine)

- Chlorines: Chlorine e6, stannous (IV) chlorine e6, chlorine e6- 2.5 N- methyl- d- glucamine (BLC1010), polylysine and polyethyleneimine conjugates of chlorine e6

- Porphyrines: HematoporphyrinHCl, photofrin, and 5- aminolevulinic acid (5- ALA), benzoporphyrin derivative (BPD)

- Xanthenes: Erythrocine

- $\quad$ Monoterpene: Azulene. ${ }^{3,5,8}$

Photofrin and hematophyrin derivatives are referred to as first generation sensitizers. Second generation photosensitizers include 5-aminolevulinic acid (ALA), benzoporphyrin derivative, texaphyrin, and temoporfin (mTHPC). These photosensitizers have greater capability to generate singlet oxygen. Third generation include Biologic conjugates (e.g. Antibody conjugate, liposome conjugate). ${ }^{10}$

\section{III .III Mechanism Of Action:}

PDT is based on the administration of a photosensitizer, which localises selectively within the target. ${ }^{19}$ The action mechanism of PDT can be briefly described as follows: upon illumination, the photosensitizer is excited and converted from the ground state to the triplet state. The longer lifetime of the triplet state enables the interaction of the excited photosensitizer with the surrounding molecules, and it is generally accepted that the generation of the cytotoxic species produced during PDT occurs while in this state. The cytotoxic product, usually singlet oxygen $\left({ }^{1} \mathrm{O} 2\right)$, has a short lifetime in biological systems $(<0.04 \mu \mathrm{s})$ and, therefore, was also shown to have a short radius of action $(0.02 \mu \mathrm{m})$. Because of the limited migration of the ${ }^{1} \mathrm{O} 2$ from the site of its formation, sites of initial cell damage from PDT are closely related to the localization of the sensitizer, thus making it ideal for local application of PDT, without endangering distant molecules, cells or organs. ${ }^{4,21,22}$ There are basically two types of reactions after onset of triplet state of photosensitizer i.e. Type I \& Type II(Fig. 4).

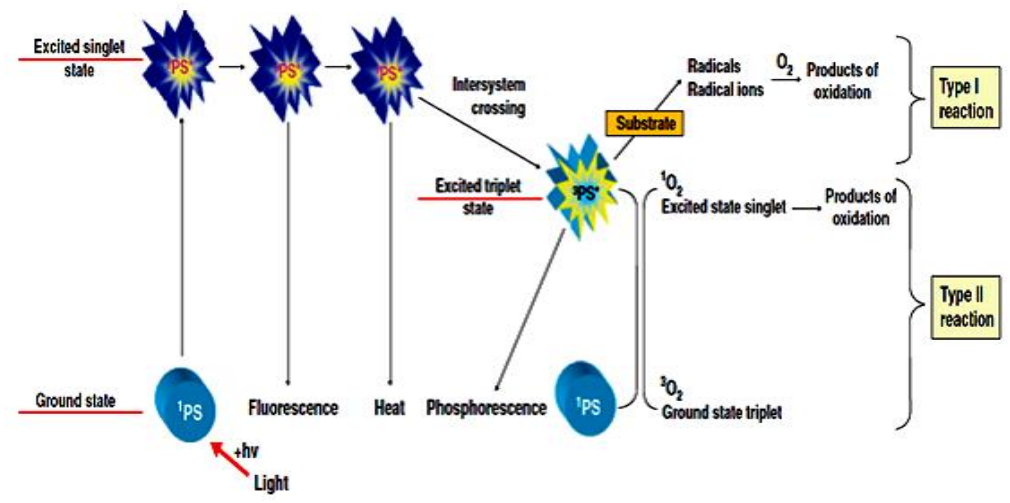

Figure 4.Type I and Type II reactions in photodynamic therapy. Following exposure to light, the activated photosensitizer (in the excited triplet state) can follow one of two pathways. The Type I pathway involves electron transfer reactions from the photosensitizer triplet state with the participation of a substrate to produce radical ions that can react with oxygen to produce cytotoxic species. The Type II pathway involves energy transfer from the photosensitizer triplet state to the ground state molecular oxygen (triplet) to produce excited state singlet oxygen, which can oxidize biological molecules. hv, photon energy; PS, photosensitizer. ${ }^{8}$ 
Type I reaction involves electron - transfer reactions between the excited state of the photosensitizer and an organic substrate molecule of the cells, producing free radicals. These free radical species are generally highly reactive and interact with endogenous molecular oxygen to produce highly reactive oxygen species, such as superoxide, hydroxyl radicals, and hydrogen peroxide, which are harmful to cell membrane integrity, causing irreparable biological damage.

In the type II reaction, the triplet-state photosensitizer reacts with oxygen to produce an electronically excited and highly reactive state of oxygen, known as singlet oxygen $\left({ }^{1} \mathrm{O} 2\right)$, which can interact with a large number of biological substrates as a result of its high chemical reactivity, inducing oxidative damage and ultimately lethal effects upon the bacterial cell bydamaging the cell membrane and cell wall. ${ }^{1}$

These photosensitizers localize in the mitochondria, plasma membrane, endoplasmic reticulum and Golgi complex at concentrations sufficient for mediating cytotoxicity. Also the reactive end products of this pathway results in a rapid cyto- and vasculotoxicity which are the basis of PDT. Research in a number of laboratories has demonstrated the potential of PDT as a treatment for localized microbial infections. PDT has shown to be active against both Gram-positive and Gram-negative organisms. ${ }^{6}$

The photodynamic activity to induce cell damage or death is determined by five important photophysical/photochemical properties including the following:

1. An overall lipophilicity and ionization of the photoreactive dyes.

2. The molecular extinction coefficient.

3. Quantum yield of the triplet state formation.

4. Redox potentials of the excited states of the $\mathrm{P}_{\text {red }}^{\mathrm{S}}$ or $\mathrm{PS}^{\mathrm{T}}{ }_{\text {red }}$, if the reaction follows the type I mechanism

5. The quantum yield of the singlet oxygen generation, if the reaction occurs by a type II photosensitization. ${ }^{6}$

\section{Application OfPdt In Periodontics And Implants}

Since the beginning of the 1990s, the application of light energy (in other words, phototherapy) has been considered as a novel treatment approach in periodontics. In general, the use of lasers has been proposed as a new technical modality in the treatment of periodontal diseases. ${ }^{23,24}$

In spite of the substantial bactericidal effects of high-level lasers ${ }^{25}$, there is limited clinical evidence to demonstrate clearly that lasers can produce a greater reduction in the number of sub-gingival bacteria than that achieved using traditional mechanical therapy. ${ }^{26}$ Also, the use of high-level lasers usually results in irreversible thermal damage to the surrounding periodontal tissues and there is a concern of unexpected side effects, such as excessive ablation or thermal coagulation, carbonization or necrosis of the root, the gingival connective tissue, the bone and the pulp tissues, depending on the type of laser employed ${ }^{27}$ Recently, a new type of noninvasive phototherapy for bacterial elimination, called photodynamic therapy, has been introduced, which uses low-level laser light. ${ }^{28}$ Unlike high-level lasers, photodynamic therapy can selectively target the bacteria without potentially damaging the host tissues. ${ }^{29}$

The photosensitizer is placed directly in the periodontal and peri-implant pocket and the liquid agent can easily access the whole root or implant surface before activation by the laser light through placement of the optical fiber directly in the pocket. As a result of the technical simplicity of the method and the high effectiveness of bacterial killing, the application of antimicrobial photodynamic therapy in the treatment of periodontal and peri-implant diseases has recently been studied extensively. ${ }^{1}$

They are also used in the treatment of oral cancer, bacterial and fungal infections, and in the photodynamic diagnosis of the malignant transformation of oral lesions. ${ }^{2,14}$ In implantology, PDT has been reported to reduce the occurrence of periimplantitis, an inflammatory process that results in infection of the surrounding tissues and leads to bone loss. As a secondary effect of its antimicrobial action, PDT can promote bone formation leading to osteointegration. ${ }^{30} \mathrm{As}$ implant placement becomes more prevalent in the treatment of edentulous areas, therapies like PDT can contribute to the long-term success of the implants. ${ }^{31}$

Photodynamic therapy has been suggested as an alternative to chemical antimicrobial agents to eliminate subgingival species and treat periodontitis. ${ }^{7}$ Antimicrobial PDT not only kills the bacteria, but may also lead to the detoxiication of endotoxins such as lipopolysaccharide. These lipopolysaccharides treated by PDT do not stimulate the production of pro-inlammatory cytokines by mononuclear cells. Thus, PDT inactivate endotoxins by decreasing their biological activity. ${ }^{2}$ Various Studies have been done regarding action of PDT on biofilms(Table. 1). 
Table 1. Photodynamic Antimicrobial Chemotherapy Studies on Plaque Biofilms in vitro

\begin{tabular}{|l|l|l|l|}
\hline Study & Photosensitizer* & Light (nm)/Laser** & Micro-organisms*** \\
\hline Dobson and Wilson (1992) & TBO, MB, AlS2Pc HP-HCl & $633 \mathrm{He} / \mathrm{Ne}$ & S.s., P.g., F.n., A.a. \\
\hline Wilson et al. (1996) & AlS2Pc & $660 \mathrm{LED}$ & S.s. \\
\hline Haas et al. (1997) & TBO & $905 \mathrm{LED}$ & A.a., P.g., P.i. \\
\hline Wood et al. (1999) & ZnPc & white light & mixed strains \\
\hline O'Neill et al. (2002) & TBO & $633 \mathrm{He} / \mathrm{Ne}$ & mixed strains \\
\hline Soukos et al. (2003) & pL-Ce6 & red light & A.n. \\
\hline Lee et al. (2004) & & $630 \mathrm{LED}$ & \\
\hline Zanin et al. (2005) & ALA & $633 \mathrm{He} / \mathrm{Ne} ; 639 \mathrm{LED}$ & P.a. \\
\hline Zanin et al. (2006) & TBO & S.m. \\
\hline Garcez et al. (2007) & TBO & $639 \mathrm{LED}$ & S.m., S.s., S.sob. \\
\hline
\end{tabular}

*AlS2Pc, aluminum disulphonatedphthalocyanine; ALA, aminolevulinic acid; $\mathrm{HP}-\mathrm{HCl}$, hematoporphyrinHCl; $\mathrm{MB}$, methylene blue; PEI-Ce6, polyethyleneimine-chlorin e6 conjugate; pL-cE6, polylysine-chlorin e6 conjugate; TBO, toluidine blue $O ; \mathrm{ZnPc}, \mathrm{Zn}(\mathrm{II})$-phthalocyanine.

**LED, light-emitting diode; He/Ne, helium/neon laser.

***A.a., Actinomycesactinomycetemcomitans; A.n., Actinomycesnaeslundii; E.f., Enterococcus faecalis; F.n., Fusobacteriumnucleatum; P.a., Pseudomonas aeruginosa; P.i., Prevotellaintermedia; P.g., Porphyromonasgingivalis; P.m., Proteus mirabilis; P.mic., Peptostreptococcus micros; S.i., Streptococcus intermedius; S.m., Streptococcus mutans; S.p., Streptococcus pyogenes; S.s., Streptococcus sanguinis; S.sob., Streptococcus sobrinus. ${ }^{3}$

Generally, antimicrobial photodynamic therapy appears to suppress periodontal pathogens and to reduce signs of inflammation effectively and safely in periodontitis in vivo. However, there is a lack of evidence to prove that antimicrobial photodynamic therapy is capable of suppressing periodontopathogens in a single dose or course. Further in vivo studies investigating the antimicrobial effects on different periodontal pathogens need to be performed. The use of antimicrobial photodynamic therapy may reduce signs of periodontal inflammation and alveolar bone loss in experimentally induced periodontitis. However, two studies have shown a tendency for regression within 30 days after treatment in the effects on bone levels. Consequently, the longterm therapeutic outcomes should be further evaluated in animal models. The limited number of in vivo studies available indicates that antimicrobial photodynamic therapy may be an alternative treatment to scaling.. ${ }^{1}$

In antimicrobial PDT, photosensitizers used are toluidine blue $\mathrm{O}$ and methylene blue. Both have similar chemical and physicochemical characteristics. Toluidine blue $\mathrm{O}$ is a solution that is blue - violet in color. It stains granules within mast cells and proteoglycans/glycosaminoglycans within connective tissues. Methylene blue is a redox indicator that is blue in an oxidizing environment and becomes colorless upon reduction. Methylene blue and toluidine blue $\mathrm{O}$ are very effective photosensitizing agents for the inactivation of both gram-positive and gram-negative periodontopathic bacteria. Tetracyclines used as antibiotics in periodontal diseases are also effective photosensitizers producing singlet oxygen. ${ }^{2,14}$

\section{IV .I Applications OfPhotoactivated Disinfection}

- PDT can be used in Non-surgical treatment of aggressive periodontitis. ${ }^{9}$

- Treating periodontal pockets.

- Plaque-infected cervical regions of teeth and implants.

- Disinfecting oral tissues prior to and during surgery.

- $\quad$ Treating oral candidiasis in immunocompromised patients. ${ }^{6}$

- Guided bone regeneration (as an adjunct in minimizing any bacterial contamination) success enhanced following Photodynamic antimicrobial chemotherapy (PACT). ${ }^{17}$

- Photodynamictherapy in implantology: Laser PDT can be used in implantology to promote osseointegration and to prevent peri- implantitis.

- $\quad$ One of the most interesting developments over the last years has been the introduction of the 9.6- $\mu \mathrm{m}$ $\mathrm{CO} 2$ laser. It has been shown in the recent literature that the use of this new device can preserve tissue, with almost no adverse effects at the light microscopic level. Intraoperatively used PDT or peri- implant care of ailing implants with the CO2 laser seems to be more of value than the conventional methods. Data suggest that lethal photosensitization may have potential in the treatment of peri- implantitis. ${ }^{5}$ 


\section{Advantages}

Benefits to be derived from the adjunctive use of PDT in providing treatment of conditions of a bacterial origin may be summarized as follows:

- Straightforward clinical technique.

- As PDT is non-invasive local therapy, following application of a sensitizer, a light source delivered into the target area precisely via a fibre optic cable, so disturbances of the microflora at other sites would not occur and damage to the adjacent host tissues can be avoided.

- PDT offers thorough irrigation and elimination of pathogens in inaccessible areas of periodontal pocket within short span of time, thus beneficial to both operator and the patient.

- The risk of bacteraemia after periodontal debridement can be minimized. Useful in patients with 'atrisk' medical history.

- There is no need to prescribe antibiotics, therefore the possibilty of side effects is avoided.

- There is no need to anaesthetize the area and destruction of bacteria is achieved in a very short period ( $<60$ seconds).

- Development of resistance to the PDT is less as singlet oxygen and other free reactive oxygen species interact with several cell structures and different metabolic pathway.

- Non-surgical protocol required for the application of the photosensitizer.

- Use as an adjunct to restorative/endodontic/surgery site pathogen reduction, PACT can disrupt plaque biofilm thus making it an adjunctive for use with ultrasonics and surfactant cleansers.

- Facilitates access into deep/limited access sites.

- Reduced need for surgery/direct flap approach. Patient comfort enhanced.

- Photodynamictherapy in implantology: Laser PDT can be used in implantology to promote osseointegration and to prevent peri-implantitis., ${ }^{4,8,17}$

\section{Drawbacks}

The risk and side effects of antimicrobial PDT are basically classified into two categories.

1. Relates to the effect of light energy.

2. Relates to the photosensitizer and the photo chemical reaction.

In photodynamic therapy, Laser power employed is very low. But during treatment procedures, irradiation of the patient's eyes must be avoided by wearing protective glasses. Most of the dyes adhere strongly to the soft tissue surface of the pocket, and retention of the dyes in the pocket, even for a short period of time, may affect periodontal tissue attachment during wound healing or may show photosensitivity towards the day light causing irritation in the area of application for the patients., ${ }^{2,14}$

\section{Perspective In Future Directions}

It is known that periodontal debridement is less effective on the biofilm in the periodontal pockets. The method of photodynamic laser therapy has contributed as an adjunctive method to the improvement of the clinical aspect of periodontal tissues and also to the reduction of bleeding on probing in the sessions following periodontal debridement. ${ }^{32}$ The technical simplicity and effective bacterial eradication are the two reasons why photodynamic therapy is extensively studied in periodontics. The concept of photodynamic laser therapy itself is very attractive because it selects the target tissue by "marking" it with the photosensitizer, and the therapy (laser energy) is active (focused) only on "marked" cells or tissues. ${ }^{5}$ The use of low-level energy lasers (i.e. diode lasers) is reported to exert additional positive effects on the surrounding tissues and cells, and they may further contribute favorably to the healing of periodontal tissues as a result of the potential biomodulatory effects, such as stimulation and proliferation of cells. ${ }^{1}$ Another important aspect of antimicrobial PDT that needs to be studied is its ability to destroy secreted virulence factors. These are substances such as lipopolysaccharides and other proteins that that may be highly vulnerable to oxidation by the ROS generated during PDT. The clinical applications of antimicrobial PDT have been slow but steady. Though limited clinical trials have been conducted for different diseases using PDT, its intensive use in periodontitis has given hope that it can be likewise used to clinically treat a number of other infectious diseases. Development of new photosensitizers, more efficient light delivery systems, and further studies are required to establish the optimum treatment parameters. ${ }^{16}$ The future of PDT will depend on the interactions between clinical applications and technological innovations. 


\section{Conclusion}

Photodynamic therapy seems to be a unique and interesting therapeutic approach toward the treatment of periodontitis and peri-implantitis. This new strategy of using PDT is less traumatic and quicker but it is still in the experimental stage of development and testing. Development of new photosensitizers, more efficient light delivery systems and further clinical studies are required to reinforce the beneficial effects of the PDT. The clinical applications of antimicrobial PDT have been slow but steady. Though limited clinical trials have been conducted for different diseases using PDT, its intensive use in periodontitis has given hope that it can be likewise used to clinically treat a number of other infectious diseases.

\section{References}

[1]. A. Takasaki, A. Aoki, K. Mizutani, F. Schwarz And A. Sculean, Periodontology 2000,51, 2009, $109-140$.

[2]. S.Rajesh,ElizabethKoshi,KoshiPhilip,AparnaMohan, Antimicrobial photodynamic therapy: An overview, Journal of Indian Society of Periodontology 15(4), 2011, 323-327.

[3]. K. Konopka1 and T. Goslinski, Photodynamic Therapy in Dentistry, Critical Reviews In Oral Biology \& Medicine, Journal of Dental Research, 86(8), 2007, 694-707.

[4]. Von Tappeiner H, JodlbauerAÜberdieWirkung der photodynamischen (fluorescierenden) Stoffe auf Protozoen und Enzyme. Dtsch Arch Klin Med 39, 1904, 427-487.

[5]. Shivakumar V., Shanmugam M., Sudhir G, PavithraPriyadarshoni S Scope of photodynamic therapy in periodontics and other fields of dentistry, Journal of Interdisciplinary Dentistry, 2(2),2012, 78-83.

[6]. Nikhil VishwasKhandge, SuchetanPradhan, YogeshDoshi, AtulKulkarni, IshanDhruva, Photodynamic Therapy (Part 1: Applications in Dentistry), International Journal of Laser Dentistry, 2013, 3(1), 7-13.

[7]. Nikolaos S. Soukos\& J. Max Goodson, Photodynamic therapy in the control of oral biofilms, Periodontology 2000, 55, 2011, 143166.

[8]. M Raghavendra, A Koregol, S Bhola, Photodynamic therapy: a targeted therapy in periodontics, Australian Dental Journa, 54(1 Suppl), 2009, S102-S109.

[9]. Rajvir Malik, AnishManocha, DK Suresh, Photodynamic therapy - A strategic review, Indian J Dent Res, 21 (2), $2010,285-291$.

[10]. Deepak Dave, Urmi Desai, NeerajDespande, Photodynamic Therapy: A View through Light, J Orofac Res 2012, 2(2), 82-86.

[11]. Dougherty TJ, Marcus SL. Photodynamic therapy. Eur J Cancer, 28, 1992, 1734-42.

[12]. Lui H, Anderson RR. Photodynamic therapy in dermatology: Shedding a different light on skin disease. Arch Dermatol, 128, 1992, 1631-6.

[13]. KamilJurczyszyn, PiotrZiółkowski, Hanna Gerber, Beata J. Osiecka, Potentiality of Photodynamic Therapy in Dentistry- Literature Review, Dent. Med. Probl,44(2)2007,255-258.

[14]. Swati Nagpal, VidyaDodwad, ShubhraVaish Photodynamic therapy:-A novel approach towards dentistry. Journal of Pharmaceutical and Biomedical Sciences (JPBMS), 21(21), 2012, 1-4.

[15]. Moan J, Peng Q. An outline of the history of PDT, in Thierry Patrice: photodynamic therapy, Comprehensive series in photochemistry and photobiology 2 . The Royal Society Of Chemistry, 2003, 1-18.

[16]. Gitika B. Kharkwal1,2, Sulbha K. Sharma1, Ying-Ying Huang1,2,3, Tianhong Dai1,2, and Michael R. Hamblin Photodynamic Therapy for Infections: Clinical Applications Lasers Surg Med, 43(7), 2011,755-767.

[17]. Steven Parker, Photodynamic antimicrobial chemotherapy in dental practice, Dental Nursing, 7(7),2011,380-385.

[18]. Allison, et al. Photosensitizers in clinical PDT. Photodiagnosis and PDT, 1, 2004, 27-42.

[19]. Marcus W. Wiedmann* and Karel Caca, General Principles of Photodynamic Therapy (PDT) and Gastrointestinal Applications, Current Pharmaceutical Biotechnology, 5, 2004,397-408.

[20]. Wainwright M. Photodynamic therapy: the development of new photosensitisers. Anticancer Agents Med Chem, 8,2008, $280-291$.

[21]. Moan J, Berg K The photodegradation of porphyrins in cells that can be used to estimate the lifetime of singlet oxygen. PhotochemPhotobiol, 53, 1991, 549-553

[22]. Peng Q, Moan J, Nesland JM Correlation of subcellular and intratumoral photosensitizer localization with ultrastructural features after photodynamic therapy. UltrastructPathol 20, 1996, 109-129.

[23]. Aoki A, Sasaki KM, Watanabe H, Ishikawa I. Lasers in nonsurgical periodontal therapy. Periodontol 2000, 36, $2004,59-97$.

[24]. Ishikawa I, Aoki A, Takasaki AA. Potential applications of Erbium:YAG laser in periodontics. J Periodontal Res, 39, 2004, 275285.

[25]. Ando Y, Aoki A, Watanabe H, Ishikawa I. Bactericidal effect of erbium YAG laser on periodontopathic bacteria. Lasers Surg Med, 19, 1996, 190-200.

[26]. Cobb CM. Lasers in periodontics: a review of the literature. J Periodontol, 77, 2006, 545-564.

[27]. Wigdor H, Abt E, Ashrafi S, Walsh JT Jr. The effect of lasers on dental hard tissues. J Am Dent Assoc 1993:124:65-70.

[28]. Wainwright M. Photodynamic antimicrobial chemotherapy (PACT). J AntimicrobChemother, 42, $1998,13-28$.

[29]. Morlock BJ, Pippin DJ, Cobb CM, Killoy WJ, Rapley JW. The effect of Nd:YAG laser exposure on root surfaces when used as an adjunct to root planing: an in vitro study. J Periodontol, 1992, 63, 1992 637-641.

[30]. Hayek RR, Araújo NS, Gioso MA et al. Comparative study between the effects of photodynamic therapy and conventional therapy on microbial reduction in ligature-Induced peri-implantitis in dogs. J Periodontol, 76(8), 2005 1275-81.

[31]. Juliana A. Barros, DDS, MS; Shalizeh A. Patel, DDS; Rachel Bishop and Ryan L Quock, Photodynamic Therapy:Shining a Light on Pathogens, Access 2012,18-19.

[32]. Mindra E. Badea, Andrea Serbanescu, MihaelaHedesiu, Alexandru F Badea, Photodynamic LasertherapyIn Patients With Periodontitis. TMJ, 60(1),2010, 18-22. 